\title{
Prognostic significance of preoperative peripheral blood neutrophil to lymphocyte ratio (NLR) in lung sarcomatoid carcinoma
}

\author{
Jun Liu ${ }^{1,2 \#}$, Shuman $\mathrm{Li}^{1 \#}$, Hao Liu ${ }^{3 \#}$, Keming $\mathrm{Chen}^{2}$, Renliu Chen ${ }^{4}$, Yongbo Xiao ${ }^{2}$, Xinke Zhang ${ }^{1,2}$, \\ Jiewei Chen ${ }^{1,2}$
}

${ }^{1}$ State Key Laboratory of Oncology in South China, Collaborative Innovation Center for Cancer Medicine, ${ }^{2}$ Department of Pathology, Sun Yat-sen University Cancer Center, Guangzhou 510060, China; ${ }^{3}$ Department of Pathology, Guangdong General Hospital's Nanhai Hospital (The Second People's Hospital of Nanhai District Foshan City), Foshan 528251, China; ${ }^{4}$ Department of Pathology, Zhuhai People's Hospital, Zhuhai 519000 , China Contributions: (I) Conception and design: J Chen, X Zhang; (II) Administrative support: Y Xiao; (III) Provision of study materials or patients: J Chen, J Liu; (IV) Collection and assembly of data: K Chen, R Chen; (V) Data analysis and interpretation: J Liu, S Li, H Liu; (VI) Manuscript writing: All authors; (VII) Final approval of manuscript: All authors.

\#These authors contributed equally to this work.

Correspondence to: Jiewei Chen. Department of Pathology, Sun Yat-sen University Cancer Center, 651\# Dong Feng Road East, Guangzhou 510060, China. Email: chenjiew@sysucc.org.cn.

Background: The present study aimed to evaluate the prognostic value of the neutrophil to lymphocyte ratio (NLR) in patients with lung sarcomatoid carcinoma (LSC).

Methods: We retrospectively analyzed the clinicopathological parameters of 100 cases of LSC in the Sun Yat-sen University Cancer Center. Receiver operator characteristics curve analysis was performed to define the cutoff point for the preoperative peripheral blood NLR. The relationship between NLR and the clinicopathological parameters of patients with LSC was estimated using $\chi^{2}$ analysis. Univariate and multivariate statistical analyses were carried out to evaluate the impact of preoperative blood NLR on postoperative survival of patients with LSC.

Results: The analyses revealed that the preoperative peripheral blood NLR was significantly associated with tumor size, $\mathrm{pN}$ stage, and clinical stage $(\mathrm{P}<0.05)$. No relationship was found between NLR and age, gender, smoking, pT stage, pM stage, relapse, and therapy. Preoperative peripheral blood NLR, clinical stage, pT stage, tumor-node-metastasis stage, and tumor size were associated with prognosis in the KaplanMeier survival analysis. Patients with a low NLR value had longer survival time than those with a high NLR [mean overall survival (OS) time: 87.1 vs. 16.0 months, $\mathrm{P}<0.05$ ]. Cox multivariate analysis confirmed that preoperative peripheral blood NLR was an independent indicator for the prognosis of LSC (hazard ratio $=3.906, \mathrm{P}<0.05)$.

Conclusions: The preoperative peripheral blood NLR was associated closely with the development and progression of LSC. Patients with an increased NLR were likely to have poor prognosis. Preoperative peripheral blood NLR might function as an important independent prognostic indicator for patients with LSC.

Keywords: Carcinoma; non-small cell lung; neutrophils; lymphocytes; prognosis; inflammation

Submitted Mar 06, 2019. Accepted for publication Aug 15, 2019.

doi: $10.21037 /$ tcr.2019.08.35

View this article at: http://dx.doi.org/10.21037/tcr.2019.08.35 


\section{Introduction}

According to the 2016 USA Cancer statistics, lung cancer ranks second for morbidity and mortality among all kinds of tumors (1). Primary sarcomatoid carcinoma of the lung is an unusual subtype of lung cancer, accounting for only $0.1-0.4 \%$ of total lung cancers (2). According to the 2015 version of World Health Organization (WHO) classification of lung cancer, LSC contains the following classifications: pleomorphic carcinoma, spindle cell carcinoma, sarcomatoid carcinoma, and pulmonary blastoma (3). Although LSC only accounts for $2.35 \%$ of non-small cell lung cancer (NSCLC), it has poor prognosis compared with other NSCLCs because of its aggressive clinical course and difficult diagnosis (4). According to a previous report, males and smokers are more susceptible to LSC.

Inflammation is considered a hallmark of cancer, contributing to its occurrence and development. Local immune responses and systemic inflammation have vital functions in the carcinogenesis and prognosis of patients with cancer (5). Recently, studies have shown that inflammation is associated with patient prognosis in many types of cancer, such as gastric cancer, NSCLC, colorectal cancer, and head and neck cancer (6-8). Many markers of inflammation, such as globulin, the neutrophil-lymphocyte ratio (NLR), the Glasgow Prognostic Score, albumin, the platelet to lymphocyte ratio, and C-reactive protein, were proven to be independent prognostic factors for cancer relapse and survival (9-11).

The NLR is a cheap, available, and reliable inflammatory marker for white blood cell analysis. The NLR often increases in inflammation because of increased neutrophils and decreased lymphocytes. The NLR can represent the state of the systemic inflammatory reaction in host immune surveillance, and an imbalance in the host immune surveillance system facilitates tumor proliferation and metastasis (12).

However, the clinical prognostic value of the NLR has not been reported in LSC. Therefore, the present study aimed to explore the relationship between the plasma NLR before therapy and the prognosis of patients with LSC.

\section{Methods}

\section{Patient specimens}

The patients with LSC who were first treated and operated on at the Cancer Center of Sun Yat-sen University from $2000 / 02$ to $2016 / 03$ were selected for this study the cases had complete clinical, pathological, and follow-up data. Routine examinations of peripheral venous blood before treatment in patients with sarcomatoid carcinoma of the lung were collected. The "neutrophil count" and "lymphocyte count" were recorded and the NLR was calculated from the absolute value of neutrophils and absolute value of lymphocytes. The diagnosis and grading of all cases in this study were based on the 2000 WHO classification criteria, according to the Cancer tumor-nodemetastasis (TNM) Classification System of 2002 version from the US Joint Commission and the International Joint Commission. Our research was authorized and approved by the Medical Ethics Committee of Sun Yat-sen University Cancer Center.

\section{Statistical analysis}

All data in our study were statistical analyzed using SPSS version 21.0 (IBM Corp., Armonk, NY, USA). Receiver operator characteristics (ROC) curve analysis was used to determine the cutoff point for the peripheral blood NLR in sarcomatoid carcinoma of the lung. The chi squared $\left(\chi^{2}\right)$ test was used to find the correlation between peripheral blood NLR and the clinicopathological features of patients with LSC. The Kaplan-Meier method was used to perform the survival analysis in sarcomatoid carcinoma of the lung, and the differences were estimated using log-rank analysis. A Cox proportional hazard regression model was carried out to perform the multivariate analyses. All the reported $\mathrm{P}$ values are two-sided. All data in this research were uploaded to the database of Sun Yat-sen University Cancer Center for future reference.

\section{Results}

\section{Characteristics of cases}

This study included 100 patients with LSC comprising 87 males (87\%) and 13 females (13\%). Their average age was 57 years old. Among them, 55 patients $(55.0 \%)$ were diagnosed at stage III and stage IV (which are considered to be late stages), while the other 45 patients $(45.0 \%)$ were diagnosed at stage I and stage II (early stages). None of the patients received any therapy before the blood test. Patients who died from reasons other than LSC were excluded from our study. Table 1 details the patients' clinicopathological features (age, gender, tumor size, TNM stage, clinical stage, and relapse). 
Table 1 Correlation between the clinicopathological variables and NLR in LSC

\begin{tabular}{|c|c|c|c|c|}
\hline Variable & \multicolumn{4}{|c|}{ NLR } \\
\hline Age (years) & & & & 0.359 \\
\hline$\leq 57^{\ddagger}$ & 47 & 25 (53.2) & $22(46.8)$ & \\
\hline$>57$ & 53 & 33 (62.3) & $20(37.7)$ & \\
\hline Male & 87 & $50(57.5)$ & 37 (42.5) & \\
\hline Female & 13 & $8(61.5)$ & $5(38.5)$ & \\
\hline Tumor size & & & & 0.017 \\
\hline$\leq 4.5^{\S}$ & 59 & $40(67.8)$ & 19 (32.2) & \\
\hline Yes & 76 & $46(60.5)$ & $30(39.5)$ & \\
\hline No & 24 & $12(50.0)$ & $12(50.0)$ & \\
\hline pT stage & & & & 0.535 \\
\hline $\mathrm{T} 1+\mathrm{T} 2$ & 56 & $34(60.7)$ & $22(39.3)$ & \\
\hline $\mathrm{T} 3+\mathrm{T} 4$ & 44 & $24(54.5)$ & $20(45.5)$ & \\
\hline pN stage & & & & 0.001 \\
\hline No & 52 & $38(73.1)$ & $14(26.9)$ & \\
\hline $\mathrm{N} 1+\mathrm{N} 2+\mathrm{N} 3$ & 48 & $20(41.7)$ & $28(58.3)$ & \\
\hline III-IV & 55 & $22(40.0)$ & $33(60.0)$ & \\
\hline Therapy" & & & & 0.701 \\
\hline Therapy 1 & 76 & $45(59.2)$ & $31(40.8)$ & \\
\hline Therapy 2 & 19 & $11(57.9)$ & $8(42.1)$ & \\
\hline Therapy 3 & 5 & $2(40.0)$ & $3(60.0)$ & \\
\hline Relapse & & & & 0.536 \\
\hline Yes & 30 & $16(53.3)$ & $14(46.7)$ & \\
\hline No & 70 & $42(60.0)$ & $28(40.0)$ & \\
\hline
\end{tabular}

${ }^{\dagger}$, Chi-square test; ${ }^{\ddagger}$, mean age; ${ }^{\S}$, mean tumor size; ", Therapy: Therapy 1 is pneumonectomy and lymphadenectomy, Therapy 2 is pneumonectomy; Therapy 3 pneumonectomy, radiotherapy, and chemoradiotherapy. NLR, neutrophil to lymphocyte ratio; LSC, lung sarcomatoid carcinoma. 

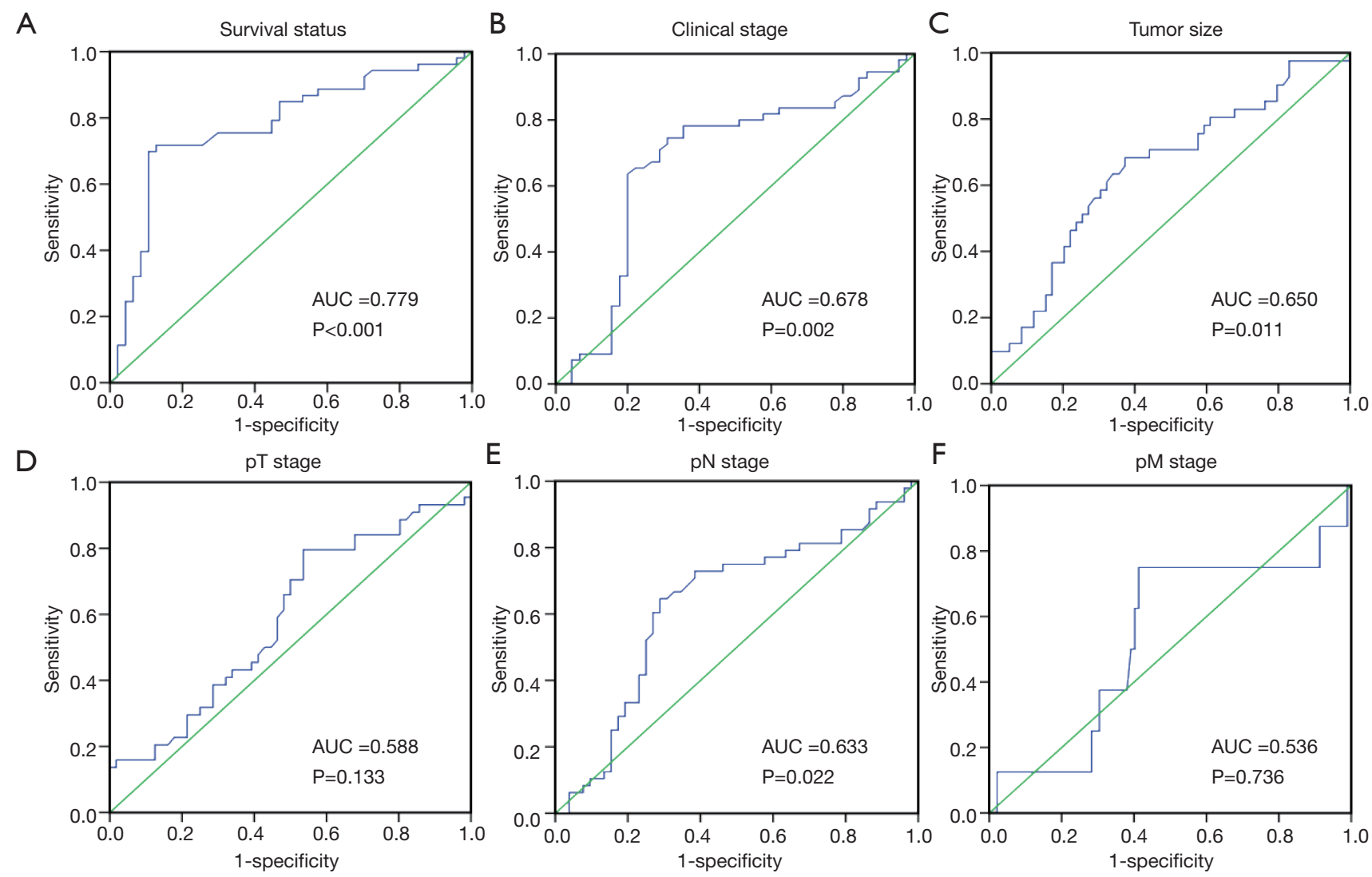

Figure 1 Receiver operator characteristics (ROC) curve analysis was employed to determine the cutoff value for the peripheral blood neutrophil to lymphocyte ratio (NLR) in lung sarcomatoid carcinoma. The sensitivity and specificity for each outcome were plotted: (A) survival status $(\mathrm{P}<0.001)$, (B) clinical stage $(\mathrm{P}=0.002),(\mathrm{C})$ tumor size $(\mathrm{P}=0.011),(\mathrm{D}) \mathrm{pT}$ stage $(\mathrm{P}=0.133),(\mathrm{E}) \mathrm{pN}$ stage $(\mathrm{P}=0.022)$ and $(\mathrm{F}) \mathrm{pM}$ stage $(\mathrm{P}=0.736)$.

\section{Selection of the cutoff value for the NLR}

ROC curves were used to analyze the NLR as status variables with survival status, clinical stage, tumor size, and TNM stage. The ROC curve was plotted with 1specific abscissa and sensitivity as the ordinate. The details are shown in Figure 1. Jordan index = sensitivity (1-specific) maximum is the best critical point, with the corresponding NLR as the cutoff value. The results of ROC curve analysis showed that the ROC curve for the NLR could be used as a survival status variable to obtain the best Jordan index, which corresponded to a NLR value of 5.2; the sensitivity and specificity of this cutoff value were 0.698 and 0.893 , respectively, with an area under the curve $(\mathrm{AUC})=0.779$.

\section{Association of the NLR with the clinicopathological features of patients with LSC}

Chi-squared tests showed that the NLR in peripheral blood was notably associated with tumor size $(\mathrm{P}=0.017)$, pN stage $(\mathrm{P}=0.001)$, and clinical stage $(\mathrm{P}<0.001)$ among the clinicopathological features. There was no significant correlation between the NLR and sex, age, smoking, pT stage, $\mathrm{pM}$ stage, therapy, and relapse (all $\mathrm{P}>0.05$ ) Table 1 .

\section{Relationships among the clinicopathological features, NLR, and patient survival: univariate survival analysis}

The clinicopathological parameters of patients with sarcomatoid carcinoma of the lung, including the NLR, 
Table 2 Univariate and multivariate analysis of neutrophil to lymphocyte ratio (NLR) associated with overall survival (OS) in patients with lung sarcomatoid carcinoma (LSC)

\begin{tabular}{|c|c|c|c|c|}
\hline Variable & \multicolumn{2}{|c|}{ Univariate analysis } & \multicolumn{2}{|c|}{ Multivariate analysis } \\
\hline Age (>57 vs. $\leq 57$ ) & $1.237(0.716-2.138)$ & 0.445 & - & - \\
\hline Gender (male vs. female) & $1.302(0.556-3.051)$ & 0.544 & - & - \\
\hline Smoking (yes vs. no) & $1.305(0.964-1.767)$ & 0.085 & - & - \\
\hline pT stage (T3 + T4 vs. T1 + T2) & $1.788(1.034-3.092)$ & 0.037 & - & - \\
\hline pN stage $(\mathrm{N} 1+\mathrm{N} 2+\mathrm{N} 3$ vs. N0) & $3.196(1.806-5.655)$ & 0.001 & - & - \\
\hline pM stage (M1 vs. M0) & $3.730(1.730-8.042)$ & 0.001 & - & - \\
\hline Clinical stage (III-IV vs. I-II) & $6.132(3.080-12.206)$ & 0.001 & $4.106(1.961-8.596)$ & 0.001 \\
\hline
\end{tabular}

$\mathrm{HR}$, hazard ratio; $\mathrm{Cl}$, confidential interval.
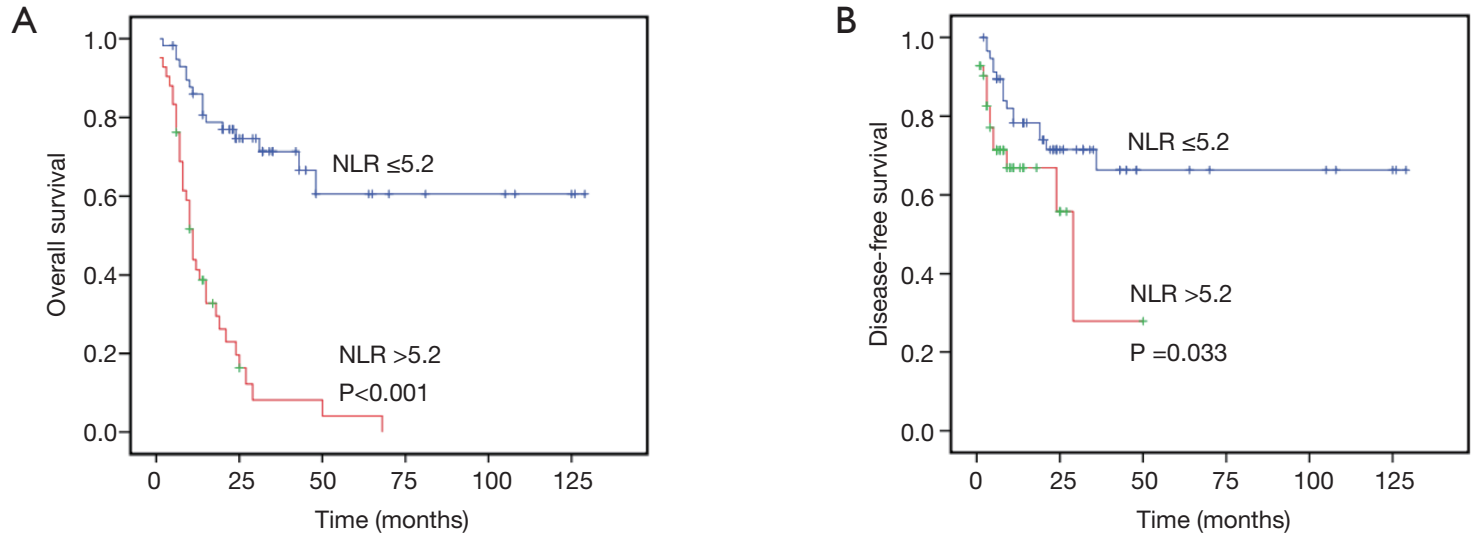

Figure 2 Kaplan-Meier analysis of OS and DFS according to the NLR in lung sarcomatoid carcinoma: A is based on OS; B is based on DFS. OS, overall survival; DFS, disease-free survival; NLR, neutrophil to lymphocyte ratio.

were analyzed using univariate analysis. The results showed that the tumor size $(\mathrm{P}=0.036)$, $\mathrm{pT}$ stage $(\mathrm{P}=0.037)$, $\mathrm{pN}$ stage $(\mathrm{P}=0.001)$, $\mathrm{pM}$ stage $(\mathrm{P}=0.001)$, clinical stage $(\mathrm{P}=0.001)$, and peripheral blood NLR $(\mathrm{P}=0.001)$ were the risk factors for the overall survival (OS) of patients with LSC. The details are shown in Table 2. Patients with a high peripheral blood NLR had an average OS of 16.0 months, which was significantly lower than the average OS of patients with a low NLR (87.1 months; $\mathrm{P}<0.001)$. The survival differences are shown in Figure $2 A$. The relationship between the peripheral blood NLR level and disease-free survival (DFS) in patients with LSC were analyzed using univariate analysis. The results indicated that the tumor size
$(\mathrm{P}=0.039)$, $\mathrm{pN}$ stage $(\mathrm{P}=0.010)$, clinical stage $(\mathrm{P}=0.002)$, and peripheral blood NLR $(\mathrm{P}=0.040)$ were risk factors for the DFS of patients with LSC. The details are shown in Table 3. Survival analysis revealed that the mean DFS was 90.28 months in patients with a low peripheral blood NLR, which was significantly higher than that of the patients with a high peripheral blood NLR (25.97 months; $\mathrm{P}=0.033)$. The survival differences are shown in Figure $2 B$.

\section{Independent prognostic factors of LSC: multivariate Cox regression analysis}

The clinicopathological parameters that were identified 
Table 3 Univariate and multivariate analysis of NLR associated with DFS in LSC patients

\begin{tabular}{|c|c|c|c|c|}
\hline Variable & \multicolumn{2}{|c|}{ Univariate analysis } & \multicolumn{2}{|c|}{ Multivariate analysis } \\
\hline Age $(>57$ vs. $\leq 57)$ & $0.684(0.332-1.409)$ & 0.303 & - & - \\
\hline Gender (male vs. female) & $1.696(0.513-5.601)$ & 0.386 & - & - \\
\hline Smoking (yes vs. no) & $1.312(0.582-2.957)$ & 0.513 & - & - \\
\hline pT stage $(\mathrm{T} 3+\mathrm{T} 4$ vs. T1 + T2) & $1.517(0.738-3.122)$ & 0.257 & - & - \\
\hline $\mathrm{pN}$ stage $(\mathrm{N} 1+\mathrm{N} 2+\mathrm{N} 3$ vs. N0) & $2.642(1.261-5.535)$ & 0.010 & - & - \\
\hline pM stage (M1 vs. M0) & $2.102(0.728-6.076)$ & 0.170 & - & - \\
\hline Clinical stage (III-IV vs. I-II) & $3.547(1.586-7.993)$ & 0.002 & $2.947(1.235-7.033)$ & 0.015 \\
\hline
\end{tabular}

as risk factors for sarcomatoid carcinoma of the lung in the univariate analysis were analyzed using a Cox regression model for multivariate analysis, The results revealed that the clinical stage [hazard ratio (HR): 4.106; 95\% confidence interval (CI): 1.961-8.596, $\mathrm{P}=0.001]$ and NLR level (HR: 3.906; 95\% CI: $2.065-7.388, \mathrm{P}=0.001$ ) could serve as independent prognostic factors for patients with sarcomatoid carcinoma of the lung. The details are shown in Table 2.

\section{Discussion}

The relationship between the systemic inflammatory response and malignant disease has been verified in previous studies. Inflammation promotes tumor development and angiogenesis, and inhibits apoptosis (13). The NLR is an inexpensive, reproducible, and widely available parameter that can be calculated during normal blood tests, which are performed routinely during a period of hospitalization. Chronic inflammation is regarded as an important factor in cancer progression. Inflammatory responses play considerable roles in the various stages of tumorigenesis, such as tumor initiation, invasion, metastasis, progression, and malignant transformation $(14,15)$. An inflammatory response provides a suitable microenvironment for tumorigenesis, development, and metastasis. Inflammation also changes the microenvironment of cancer by altering stromal cell turnover rates and affecting the immunosuppressive capabilities of immune cells (16).

Neutrophils produced during the inflammation response are key mediators of cancer progression, providing the cancer microenvironment with bioactive molecules and inducing the development of other hallmarks of cancer, such as genetic instability and angiogenesis. In addition, the increasing number of neutrophils around cancer tissues can suppress activated $\mathrm{T}$ cells and natural killer cells, which could exert an antitumor immune response (17). The NLR, as an index of systemic inflammation, is a proven prognostic indicator for many types of solid cancer, such as urinary cancer, colorectal cancer, intraductal papillary mucinous neoplasms of the pancreas and gastroesophageal junction, and gastric cancer (18-21).

A meta-analysis of the NLR in gastric cancer, which included 19 studies with 5,431 patients, concluded that an elevated NLR correlated with a significantly poorer outcome in terms of OS and progression-free survival (22). Another systematic review of the NLR in urothelial cancer, which studied 6,240 patients with urothelial cancers, found that the NLR was deemed to be a worse prognostic biomarker in $87.5 \%$ of upper tract urothelial cancer, $80 \%$ of urothelial bladder cancer, and $60 \%$ of metastatic and advanced disease (23). These studies suggested that the NLR could be used as a biomarker for cancer. Researchers have studied NLR in patients with resected lung cancer, and the results revealed that an increasing NLR was associated with higher stage and was an independent risk factor for reduced survival after resection for primary lung cancer $(24,25)$; therefore, we hypothesized that the NLR could be used as a biomarker for LSC.

The present study comprised a retrospective analysis of the preoperative NLR level in 100 cases of LSC. The result showed that the preoperative peripheral blood NLR was notably associated with tumor size, $\mathrm{pN}$ stage, and clinical 
stage. Univariate analysis revealed that the tumor size, pT stage, pN stage, pM stage, clinical stage, and peripheral blood NLR were risk factors for LSC. Multivariate analysis revealed that the clinical stage and peripheral blood NLR were independent prognostic factors for LSC. These results showed that NLR could be used as a prognosis biomarker for LSC, which was consistent with the above-mentioned studies.

The association between an increased NLR and poor outcome of cancer has been well studied; however, the mechanism of this effect and how to apply the NLR clinically, require further research. Neutrophils have been shown to suppress the immune system and T-cell response (26), which could be used as a predictor of clinical responses to immunotherapy, such as PD-L1 (programmed cell death 1 ligand 1) and PD-1 (programmed cell death 1). Clinical research focused on the peripheral blood NLR before and after the immunotherapy might show that NLR in combination with other prognostic markers could identify patients in whom treatment would be more effective. The cancer prevention effects of anti-inflammatory drugs, such as aspirin and other non-steroid anti-inflammatory drugs (NSAIDs), as a primary cancer prevention method, remains controversial and may be more beneficial in certain cancers (27). The administration of NSAIDs as part of the postoperative management of lung cancer after surgery showed that anti-inflammatory drugs were marginally associated with better OS (28). However, the regular use of aspirin causes more bleeding; therefore, the estimate of the inflammation level (such as an increased NLR) before the use of anti-inflammatory drugs will be more important.

There were some limitations in the design and implementation of this study. This was a retrospective study comprising only 100 cases of LSC collected from one center. Nevertheless, this is the first report of the prognostic significance of the NLR in LSC, and further multi-institutional investigations are warranted to define the prognostic implication of the NLR in LSC.

In conclusion, the results of the present study suggested that the preoperative peripheral blood NLR was significantly related to carcinogenesis and progression of LSC. The preoperative peripheral blood NLR could function as an independent prognostic factor for patients with LSC.

\section{Acknowledgments}

Funding: This study was supported by the Youth Foundation of National Natural Science Foundation of China (81702755).

\section{Footnote}

Conflicts of Interest: All authors have completed the ICMJE uniform disclosure form (available at http://dx.doi. org/10.21037/tcr.2019.08.35). The authors have no conflicts of interest to declare.

Ethical Statement: The authors are accountable for all aspects of the work in ensuring that questions related to the accuracy or integrity of any part of the work are appropriately investigated and resolved. The study was conducted in accordance with the Declaration of Helsinki (as revised in 2013). The study was approved by the Institute Research Medical Ethics Committee of Sun Yat-sen University Cancer Center (No. YB2018-016). Individual informed consent was waived.

Open Access Statement: This is an Open Access article distributed in accordance with the Creative Commons Attribution-NonCommercial-NoDerivs 4.0 International License (CC BY-NC-ND 4.0), which permits the noncommercial replication and distribution of the article with the strict proviso that no changes or edits are made and the original work is properly cited (including links to both the formal publication through the relevant DOI and the license). See: https://creativecommons.org/licenses/by-nc-nd/4.0/.

\section{References}

1. Siegel RL, Miller KD, Jemal A. Cancer statistics, 2016. CA Cancer J Clin 2016;66:7-30.

2. Yendamuri S, Caty L, Pine M, et al. Outcomes of sarcomatoid carcinoma of the lung: a Surveillance, Epidemiology, and End Results Database analysis. Surgery 2012;152:397-402

3. Travis WD, Brambilla E, Nicholson AG, et al. The 2015 World Health Organization Classification of Lung Tumors: Impact of Genetic, Clinical and Radiologic Advances Since the 2004 Classification. J Thorac Oncol 2015;10:1243-60.

4. Park JS, Lee Y, Han J, et al. Clinicopathologic outcomes of curative resection for sarcomatoid carcinoma of the lung. Oncology 2011;81:206-13.

5. Diakos CI, Charles KA, McMillan DC, et al. Cancerrelated inflammation and treatment effectiveness. Lancet 
Oncol 2014;15:e493-503.

6. Scott HR, McMillan DC, Forrest LM, et al. The systemic inflammatory response, weight loss, performance status and survival in patients with inoperable non-small cell lung cancer. Br J Cancer 2002;87:264-7.

7. Gao Y, Huang D. The value of the systematic inflammation-based Glasgow Prognostic Score in patients with gastric cancer: a literature review. J Cancer Res Ther 2014;10:799-804.

8. Rassouli A, Saliba J, Castano R, et al. Systemic inflammatory markers as independent prognosticators of head and neck squamous cell carcinoma. Head Neck 2015;37:103-10.

9. Wang D, Yang JX, Cao DY, et al. Preoperative neutrophillymphocyte and platelet-lymphocyte ratios as independent predictors of cervical stromal involvement in surgically treated endometrioid adenocarcinoma. Onco Targets Ther 2013;6:211-6.

10. Yodying H, Matsuda A, Miyashita M, et al. Prognostic Significance of Neutrophil-to-Lymphocyte Ratio and Platelet-to-Lymphocyte Ratio in Oncologic Outcomes of Esophageal Cancer: A Systematic Review and Metaanalysis. Ann Surg Oncol 2016;23:646-54.

11. Xin-Ji Z, Yong-Gang L, Xiao-Jun S, et al. The prognostic role of neutrophils to lymphocytes ratio and platelet count in gastric cancer: A meta-analysis. Int J Surg 2015;21:84-91.

12. Vesely MD, Schreiber RD. Cancer immunoediting: antigens, mechanisms, and implications to cancer immunotherapy. Ann N Y Acad Sci 2013;1284:1-5.

13. Coussens LM, Werb Z. Inflammation and cancer. Nature 2002;420:860-7.

14. Grivennikov SI, Greten FR, Karin M. Immunity, inflammation, and cancer. Cell 2010;140:883-99.

15. Wiig H, Swartz MA. Interstitial fluid and lymph formation and transport: physiological regulation and roles in inflammation and cancer. Physiol Rev 2012;92:1005-60.

16. Hanahan D, Weinberg RA. Hallmarks of cancer: the next generation. Cell 2011;144:646-74.

17. Zhu H, Cao X. NLR members in inflammation-associated carcinogenesis. Cell Mol Immunol 2017;14:403-5.

18. Wei Y, Jiang YZ, Qian WH. Prognostic role of NLR in urinary cancers: a meta-analysis. PLoS One 2014;9:e92079.

19. Li MX, Liu XM, Zhang XF, et al. Prognostic role of neutrophil-to-lymphocyte ratio in colorectal cancer: a systematic review and meta-analysis. Int J Cancer
2014;134:2403-13.

20. Wang SC, Chou JF, Strong VE, et al. Pretreatment Neutrophil to Lymphocyte Ratio Independently Predicts Disease-specific Survival in Resectable Gastroesophageal Junction and Gastric Adenocarcinoma. Ann Surg 2016;263:292-7.

21. Gemenetzis G, Bagante F, Griffin JF, et al. Neutrophilto-lymphocyte Ratio is a Predictive Marker for Invasive Malignancy in Intraductal Papillary Mucinous Neoplasms of the Pancreas. Ann Surg 2017;266:339-45.

22. Sun J, Chen X, Gao P, et al. Can the Neutrophil to Lymphocyte Ratio Be Used to Determine Gastric Cancer Treatment Outcomes? A Systematic Review and MetaAnalysis. Dis Markers 2016;2016:7862469.

23. Marchioni M, Primiceri G, Ingrosso $M$, et al. The Clinical Use of the Neutrophil to Lymphocyte Ratio (NLR) in Urothelial Cancer: A Systematic Review. Clin Genitourin Cancer 2016;14:473-84.

24. Tomita M, Shimizu T, Ayabe T, et al. Preoperative neutrophil to lymphocyte ratio as a prognostic predictor after curative resection for non-small cell lung cancer. Anticancer Res 2011;31:2995-8.

25. Sarraf KM, Belcher E, Raevsky E, et al. Neutrophil/ lymphocyte ratio and its association with survival after complete resection in non-small cell lung cancer. J Thorac Cardiovasc Surg 2009;137:425-8.

26. De Larco JE, Wuertz BR, Furcht LT. The potential role of neutrophils in promoting the metastatic phenotype of tumors releasing interleukin-8. Clin Cancer Res 2004;10:4895-900.

27. Rostom A, Dube C, Lewin G, et al. Nonsteroidal antiinflammatory drugs and cyclooxygenase- 2 inhibitors for primary prevention of colorectal cancer: a systematic review prepared for the U.S. Preventive Services Task Force. Ann Intern Med 2007;146:376-89.

28. Choi JE, Villarreal J, Lasala J, et al. Perioperative neutrophil:lymphocyte ratio and postoperative NSAID use as predictors of survival after lung cancer surgery: a retrospective study. Cancer Med 2015;4:825-33.

Cite this article as: Liu J, Li S, Liu H, Chen K, Chen R, Xiao Y, Zhang X, Chen J. Prognostic significance of preoperative peripheral blood neutrophil to lymphocyte ratio (NLR) in lung sarcomatoid carcinoma. Transl Cancer Res 2019;8(5):1826-1833. doi: $10.21037 /$ tcr.2019.08.35 